\title{
Freeform Shape Clustering For Customized Design Automation
}

\author{
Alexander Zouhar \\ Siemens Corporate Research, Inc. \\ Princeton, NJ \\ alexander.zouharesiemens.com \\ Sajjad Baloch
}

Gozde Unal Tong Fang
Sergei Azernikov Claus Bahlmann

\author{
Siegfried Fuchs \\ Dresden University of Technology \\ Institute of Artificial Intelligence \\ siegfried.fuchseinf.tu-dresden.de
}

\begin{abstract}
Automation can provide significant performance improvements in digital manufacturing systems that customize shapes of implants and prosthetic devices to the anatomy of a patient. The challenge, however, lies in the ability of an automatic solution to adapt to anatomical variations of a given object category. This paper presents a hierarchical framework that generalizes the digital design of anatomical surface models in terms of a small number of prototypes. The latter are derived from the local shape information of constituent parts via shape matching and clustering and then associated with one operation that dictates how a shape undergoes modification. We demonstrate the proposed technique through application to typical hearing aid design operations with promising results.
\end{abstract}

\section{Introduction}

Automatic personalized design of anatomical surface models is a challenging problem. It leads to a reduction in the amount of manual work that is needed to modify a shape in such a way that it comfortably fits the anatomy of a patient. This is highly significant for applications related to mass customization of medical devices, such as hearing aids, implants, and prosthetic devices [1, 2, 3, 4].

Current practice of hearing aid design involves a sequence of surface modifications that are carried out manually by an operator using a CAD software system. Input to the process is a surface mesh, which is obtained through scanning and surface reconstruction of an ear-mold taken from the ear canal and external ear of a patient. The reconstructed surface is a triangle mesh approximating a 2D manifold embedded in $\mathbb{R}^{3}$. Work instructions describe where, for instance, in terms of anatomical landmarks, and how the mesh geometry should be modified. The requirement is to define a cutting plane, after which some additional CAD process is employed to modify a surface mesh locally (for instance, via cutting, smoothing, deformation, or a combination thereof ${ }^{1}$ ). The eventual end product constitutes the shape of a hearing aid (HA) device, customized for the person the mold has been taken from (please refer to $[1,17]$ for more details about digital HA design). Subsequently, we use the more comprehensive term of an operation, which includes the definition of a cutting plane and the intended surface modification.

Inadequacies of operators, missing repeatability, and lack of efficiency are contributing factors for high recall rates, forcing manufacturers to spend large amounts of their resources on remakes. Consequently, the principle idea of semi-automatic or even fully automatic shape design is appealing for improving the production performance (product quality, manufacturing time, customer satisfaction).

For increasing efficiency, repeatability and patient comfort, automatically modifying an anatomical surface mesh in a prescribed way to yield the customized product is desirable. The challenge, however, lies in the ability of an automatic solution to adapt to anatomical variations of a given object category.

A fully automatic solution to customized shape design has recently been proposed in [5]. It models the relationship between two classes of shapes as a mapping based on multivariate linear regression, where principal component analysis (PCA) is used to capture the variation across indi-

\footnotetext{
${ }^{1}$ In some cases a mesh modification may require more than one cutting plane in order to define a sufficient local constraint. For instance, one may define two cutting planes, where one of the planes is defined relative to the first one. The region between the two planes is then modified, for instance via smoothing or deformation.
} 
vidual instances. The main issue with this approach is its lack of interpretability, which often becomes problematic when results deviate from the expected outcome.

Our work is similar in spirit to the manufacturing classification problem of CAD models in [6], where a methodology for discriminating between two machining processes of mechanical artifacts was presented. However, our aim is to identify an unknown number of "machining processes", each of which will define a certain set of rules for a specific CAD operation.

\subsection{Our contribution}

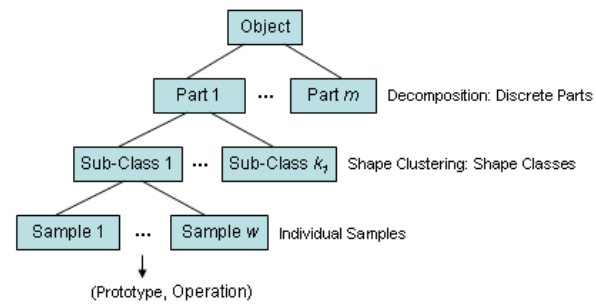

Figure 1. Hierarchical framework for the generation of prototypes for automatic, customized shape design. The number of subclasses/prototypes $k_{l}$ may differ among the part categories, which are indicated by the subscript $l \leq m$. An operation when assigned to a prototype forms a processing template.

Our approach, herein, is to generalize customized shape design via a small number of prototypical shapes. Each prototypical shape is associated with one operation that defines how similar shapes undergo modifications. Anatomical variations may require that the position of a cutting plane is adapted to the anatomical peculiarities of individual instances in order to obtain an acceptable result.

To this end, we consider an object as an union of discrete parts as illustrated in Fig. 1. For a pending design procedure, relevant parts of objects of a given category are clustered into a small number of sub-classes according to their shape distances, where the number of clusters is unknown prior to clustering. The variability within each subclass is then compactly represented by a characteristic class member, which we refer to as prototype. If no distinct clusters exist, the prototype generation should at least provide a reasonable down sampling of the set of possible shapes. Finally, an expert assigns one operation to each prototype by defining a cutting plane that intersects the surface part, according to its category and its shape. When assigned to a prototype, an operation forms a processing template.

Given an input surface and its decomposition, we propose a customized design approach which consists of: (1) finding the best-matching prototype for each of its constituent parts; (2) inferring the operations from the prototypes to the corresponding input parts (e.g., by propagating a cutting plane to an input part using a geometric transfor- mation relating the prototype to the input part); and (3) automatically modifying a shape by using a CAD process of the manufacturing software. This potentially allows manual corrections (e.g., via rotation and translation of a cutting plane) prior to surface modification, which removes the major limitation of a fully automatic approach that would estimate the final shape without taking into account the specifics of the CAD process.

The decomposition of objects into parts forms a powerful ingredient of our approach. It enables the system to discard confounding influences from less relevant parts, and to focus on more pertinent regions. This facilitates more accurate inferences on operations from the prototypes to new instances. For example, in the design of a dental implant, the instruction "smooth sharp edges at the crown" imposes a spatial constraint on a smoothing operation, excluding unrelated parts. One of the main contributions of our work is to lift the necessity of defining a cutting plane on every shape during rapid prototyping.

We demonstrate the proposed technique on two typical operations of digital hearing aid design with promising results. The next Section provides an overview of the proposed technique, followed by the framework for shape matching (Section 2.1), clustering (Section 2.2), and operation inference (Section 2.3), which form the main building blocks of our approach. Experimental results are presented in Section 3. A final discussion concludes our work.

\section{Method}

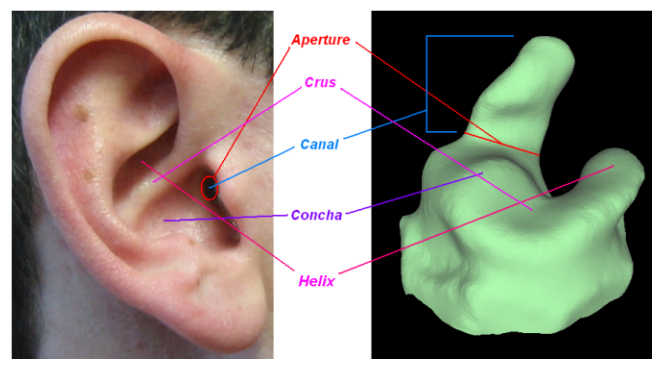

Figure 2. Important anatomical features of the human ear. The decomposition of a surface mesh (Right) into canal part and external ear part is defined by the aperture contour.

Surface decomposition. The decomposition of a surface into parts is process related and is anatomically founded. It shall be constrained in such a way that a modification of one part has no confounding effect on other parts. In this work, we use the algorithm explained in [16] to automatically decompose a surface mesh of an ear-mold into canal part and external ear part. The decomposition is defined by the aperture contour, which is shown in Fig. 2 among other important features of the human ear ${ }^{2}$.

\footnotetext{
${ }^{2}$ By courtesy of the authors of [16].
} 


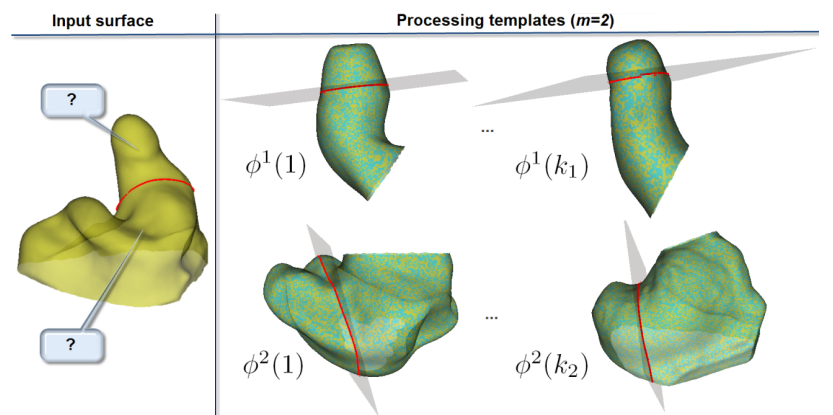

Figure 3. Illustration of the proposed method with application to HA design. Shown are processing templates with predefined cutting planes for two part categories $(m=2)$, i.e., canal $(l=1)$ and external ear $(l=2)$. There are $k_{1}$ templates for parts of category $l=1$ and $k_{2}$ templates for parts of category $l=2$. An input surface is decomposed into two parts via the aperture contour which is depicted in red color (cf. Fig. 2). The resulting task is to determine the best-matching processing template $\phi^{l}(\cdot)$ for each input part as indicated by the question marks. All surfaces are transparently rendered.

Clustering of categorical parts. Given a training set of surface meshes and their decompositions into $m$ parts, we devise a clustering procedure that finds a (natural) grouping of parts of the same category with label $l \leq m$, according to their shape distances. Each shape cluster is then compactly represented by a cluster member, which we refer to as prototype.

Operation assignment. Subsequently, an operation is assigned to each prototype by a process engineer. As stated earlier, such operations dictate how a shape undergoes modification. The assignment of an operation is conducted by defining a cutting plane on the surface mesh according to its shape. Together, a prototype and its assigned operation form a processing template for parts of category $l \leq m$, say template $\phi^{l}$.

This results in a set of possible processing templates for each part category $l \leq m$, i.e., $\Phi^{l}=\left\{\phi^{l}(j): 1 \leq j \leq\right.$ $\left.k_{l}\right\}$, where $k_{l}$ denotes the number of recognized prototypes. Notice, that the number of prototypes $k_{l}$ may differ among the categories, which are indicated by the subscript $l$. The set of prototypical design processes, say $\Theta$ is, hence, given by the product set $\Theta=\Phi^{1} \times \cdots \times \Phi^{m}$. Fig. 3 illustrates the resulting task for an input surface.

Resulting task. The task for a new input surface is to find a sufficient design process $\theta^{*} \in \Theta$ that yields the customized product. To do so, we infer the operation for each part of an input surface from the best-matching candidate prototype, (e.g., by propagating the cutting plane using a geometric transformation relating the prototype to the input part). Operations are then carried out automatically by employing a deterministic CAD process of the manufacturing software that is normally used interactively.

\subsection{Shape matching and distance of parts}

A meaningful notion of shape distance is often coupled to the problem of establishing correspondences between points of similar object parts. Further requirements include symmetry, invariance to group actions (e.g. affine), and robustness to non-ideal conditions, which may arise from noisy 3D scans and small local deformations. This requires rich local descriptors for estimating point correspondences.

For simplicity and tractability, we prefer to sample points from a surface with roughly uniform spacing. This is done by embedding a surface mesh, say $X$ or $X^{\prime}$ in a bounding cube that is subdivided into uniform bins. Inside each nonempty bin we pick the nearest (in terms of the Euclidean distance) surface point to the center of the bin. To ensure invariance of point sampling with respect to rigid transformations of a surface, the orientation of the bounding cube is defined via PCA of the mesh vertex coordinates. Furthermore, the bin size of the bounding cube must be chosen carefully in order to preserve informative surface features.

The proposed sampling technique generally leads to unequal cardinality point sets, i.e., $\left|P_{l}\right| \neq\left|P_{l}^{\prime}\right|, P_{l} \subset X$, $P_{l}^{\prime} \subset X^{\prime}$. In this setting, a shape distance relates to the partial matching of the two point sets. In the subsequent discussion we consider parts of the same category and omit the index $l$.

We express point correspondence as a one-to-one mapping $f: I \rightarrow J$ between sampled point sets $P \subset X, P^{\prime} \subset X^{\prime}$, where $I=\left\{i \in \mathbb{N}: p_{i} \in P\right\}$ and $J=\left\{j \in \mathbb{N}: p_{j} \in P^{\prime}\right\}$ denote the (unordered) index sets of $P$ and $P^{\prime}$. Without loss of generality, we assume that $|I| \leq|J|$. The optimal correspondence $f^{*}$ is obtained by minimizing, for example, the sum of local matching costs $q(\cdot, \cdot)$, i.e.,

$$
Q(f)=\sum_{i \in I} q\left(p_{i}, p_{f(i)}^{\prime}\right)
$$

As a solution, we extend the shape context (SC) framework in [8] to 3D, which in contrast to [9] and [11] finds an optimal correspondence $f^{*}$ subject to the constraint that the mapping is one-to-one. This requires a square cost matrix which is obtained by adding a constant high matching cost to the rows and/or columns. Like in [8], we define $q(\cdot, \cdot)$ as the $\chi^{2}$ test statistic of the normalized SC histograms at $p_{i}$ and $p_{f(i)}^{\prime}$. Given the point-by-point scores $q(\cdot, \cdot)$ the precise set of matches is then obtained via bipartite graph matching using the algorithm in [14]. We note that in contrast to our approach, the iterative closest point algorithm (ICP) [7] is known to generate a lot of local minima when assigning correspondences and does usually not guarantee that the correspondences are one-to-one.

Given the current best correspondence $f^{*}$, we proceed to find the parameters of a transformation $T_{P P^{\prime}}: \mathbb{R}^{3} \rightarrow \mathbb{R}^{3}$ chosen from a suitable family, which, when applied to $P$, 
best aligns it with $P^{\prime}$ by minimizing

$$
E\left(T_{P P^{\prime}}\right)=\sum_{i \in I}\left[p_{j}^{\prime}-T_{P P^{\prime}}\left(p_{i}\right)\right]^{T} W_{i}\left[p_{j}^{\prime}-T_{P P^{\prime}}\left(p_{i}\right)\right],
$$

where $j=f^{*}(i)$. The weight matrix $W_{i} \in \mathbb{R}^{3} \times \mathbb{R}^{3}$ encodes additional prior knowledge about each correspondence pair $\left(p_{i}, p_{j}^{\prime}\right)$, in the form of a level of confidence. For instance, for an isotropic case, $W_{i}$ reduces to $w_{i} \mathbf{I}$. In general, a suitable $w_{i}$ may readily be specified by noting that local mismatches between points $p_{i} \in P$ and $p_{j}^{\prime} \in P^{\prime}$ tend to increase the cost of their correspondence. Enforcing uniqueness of matching may lead to situations where a point in $P$ gets assigned to a point in $P^{\prime}$ at a higher local cost $q(\cdot, \cdot)$ compared to the nearest neighbor assignment. In order to improve the quality of the transformation estimate, we set $w_{i}$ to

$$
w_{i}=\frac{1}{Z_{i}} \exp \left(-\left[z_{i}-z_{i}^{*}\right]^{2}\right)
$$

where $z_{i}=q\left(p_{i}, p_{j}^{\prime}\right)$ denotes the local cost of matching $p_{i}$ and $p_{j}^{\prime}$ according to $f^{*}$, and $z_{i}^{*}$ indicates the actual minimal cost of matching $p_{i}$ to some point $p^{\prime} \in P^{\prime}$, which is not necessarily $p_{j}^{\prime} \in P^{\prime}$. $Z_{i}$ denotes the integration constant. The value of $w_{i}$ reduces the influence of locally unfavorable matches during the estimation of $T_{P P^{\prime}}$. In addition, points that are assigned to one of the added rows/columns are treated as outliers and are discarded from the computation of $E\left(T_{P P^{\prime}}\right)$ in Eqn. (2).

The sequence of correspondence estimation and alignment may be iterated in order to improve their combined performance. After convergence the sum of the final shape context matching costs is considered as directed distance $D_{P P^{\prime}}$ between two point sets $P, P^{\prime}$, i.e.,

$$
D_{P P^{\prime}}=\frac{1}{M} \sum_{i \in I} q\left(T_{P P^{\prime}}\left(p_{i}\right), p_{j}^{\prime}\right),
$$

where $j=f^{*}(i)$ and $M$ denotes the number of participating point pairs. We measure the shape distance between two parts of $X$ and $X^{\prime}$ as the average of their directed distances, i.e.,

$$
\bar{D}=\frac{\left(D_{P P^{\prime}}+D_{P^{\prime} P}\right)}{2}
$$

where $\bar{D}$ is symmetric.

The employed metric was found to yield excellent clustering/separation within a class of shapes, as we are interested in subtle variations in anatomy in the form of say bends. It effectively allowed highlighting changes we were after. However, several descriptor choices are available for this step, for example, spin images [10], variable dimensional local shape descriptors [12], relative-angle context distributions [13] to mention a view. See also the references in [12]. In order to develop the different stages of our approach, we allow abstraction from the concrete choice of the descriptor.

\subsection{Shape clustering and prototype selection}

Equipped with the notion of shape distance we proceed with the unsupervised grouping of $n$ categorical parts into $k_{l}$ clusters, where $l$ is fixed. In general, the solution to the problem is obtained by optimizing an objective function that depends on $n$ hidden labels corresponding to $n$ data samples, where the label $c_{i} \in\left\{1, \ldots, k_{l}\right\}, i \leq n$ denotes the class association of data sample $i$.

We use Affinity Propagation (AP) [15] to cluster the shapes, while simultaneously identifying exemplars that best represent other cluster members. The algorithm takes general nonmetric similarities as an input and determines the number of clusters depending on a weight that indicates how likely it is for each data sample to be chosen as an exemplar. A common weight for all training data indicates a priori that they are equally suitable as exemplars.

AP interprets each data sample as a node in a network. The algorithm then recursively exchanges two competing types of real valued messages between the data samples in order to determine which samples are exemplars and to which exemplar every other sample is assigned. AP maximizes an energy function over all valid configurations $c=\left(c_{1}, \ldots, c_{n}\right)$, called net similarity which is given by $E(c)=\sum_{i=1}^{n} s\left(i, c_{i}\right)$, where $s\left(i, c_{i}\right)$ is the similarity of data sample $i$ to its exemplar. Therein, the weights appear as values $s(i, i)$, i.e., $c_{i}=i$.

Compared to related techniques, AP is not susceptible to poor initializations and does not lead to solutions that may result from hard decisions, for example by storing a relatively small number of estimated cluster centers at each step ( $k$-means, Expectation Maximization (EM) algorithm).

When the number of clusters is not apparent from prior knowledge, AP can be executed several times with weight values that relate to exactly $k_{l}$ clusters. A standard approach for estimating the optimal number of clusters is to plot the clustering error against the number of clusters. The latter is then chosen at the point where the clustering error starts to decrease more slowly, which we will show in Section 3. We use the resulting set of $k_{l}$ exemplars as prototypes.

\subsection{Inferring operations using prototypes}

After shape clustering one particular operation is assigned to each part prototype by an expert as stated earlier. The part prototypes may now be utilized for automatic, customized shape design.

Fig. 4 illustrates the principle idea on the example of two typical HA design operations, (1) canal modification, (2) helix modification. Both operations require the definition of a cutting plane on each of two constituent parts (blue shaded) according to their shape, (1) the canal part, and (2) the external ear part. Fig. 2 depicts several anatomical features and parts of the human ear including the canal part and 

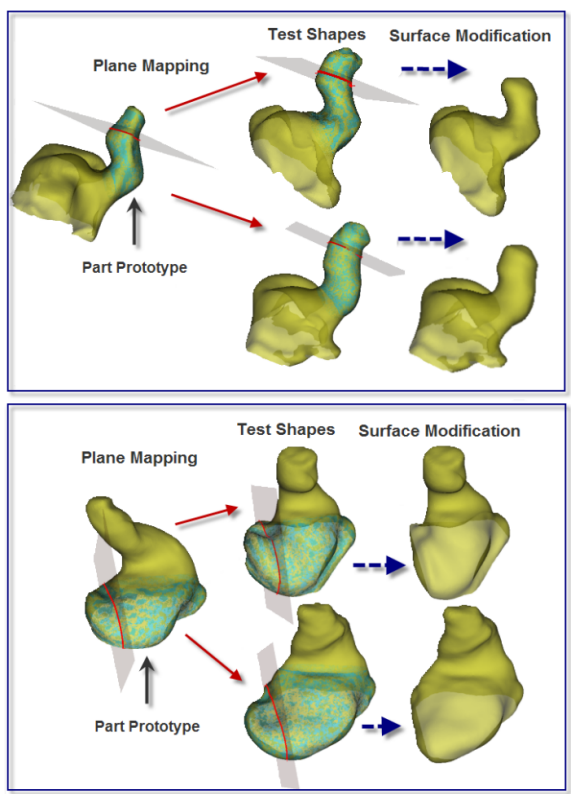

Figure 4. Inferring operations using prototypes in HA design. Each frame shows the best-matching prototype (Left) for two test shapes (Middle) with respect to the blue shaded part. The intersection plane on the prototype specifies an operation. The plane is propagated from the prototype to the corresponding part of each test shape. Executing the operation yields the modified surface (Right). All surfaces are transparently rendered.

the external ear part. Notice, that the helix is a sub-region of the external ear part.

Each plane implicitly defines a simple, planar contour (red) that marks the support region of a deterministic CAD process. A plane (planar contour) is then propagated from the best-matching prototype to the test shape by applying the geometric transformation $T_{P P^{\prime}}$ which has been estimated using Eqn. (2) relating the prototype to the test shape. If $T_{P P^{\prime}}$ is chosen to be nonlinear, then a plane could simply be refit to a propagated contour, even if the warped contour points do not lie on the target surface.

Our work here focuses on the accurate placement of a cutting plane around a certain anatomical landmark as presented above for the canal part and the external ear part. Following this crucial step, a standard mesh modification is carried out on one side of the plane by a CAD process, similar to those available in various commercial packages $[18,19,20]$. The output of our proposed method as a result is a modified mesh geometry, which is customized to a given patient, however following certain operational rules of the given design process.

\section{Results}

We acquired a training set of 184 ear anatomy mesh models which were obtained from hearing impaired individ-
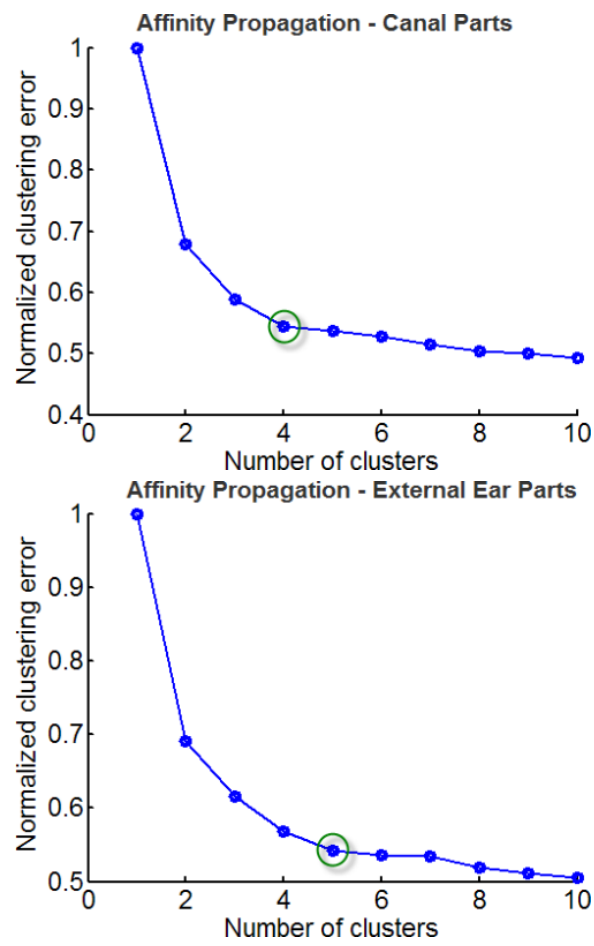

Figure 5. Normalized clustering error as a function of the number of clusters. The clustering error is the negative net similarity for $k_{l}$ clusters after convergence, normalized by the error value for clustering with $k_{l}=1$. The gain in information drops significantly after (Top) $k_{1}=4$ classes and (Bottom) $k_{2}=5$ classes.

uals as explained in Section 1. We demonstrate our framework on two typical operations of digital hearing aid design (1) canal modification, (2) helix modification. Both operations require the positioning of a cutting plane on each of two constituent parts, (1) the canal part, and (2) the external ear part. Each surface was first segmented into canal part $(l=1)$, and external ear part $(l=2)$. As stated earlier we employed the method described in [16] for this purpose.

Both training data sets were clustered separately after point sampling and computation of pairwise distances using an affine transformation model. For point sampling a bin size of 0.02 units was found to be sufficient assuming that the bounding cube has unit length in each dimension. The number of clusters was determined by examining the graphs in Fig. 5. We conclude that the training set of canal parts is reasonably well represented by four clusters/prototypes (different degrees of canal bending), and the training set of external ear parts by five clusters/prototypes.

A comparison of Fig. 6(a) with Figs. 6 (b)-(e) shows that residual shape differences become increasingly localized, while concurrently the local variances decrease for the remaining surface. Similar observations can be made for the training set of external ear parts by comparing Fig. 7 (a) with Figs. 7 (b)-(f). 


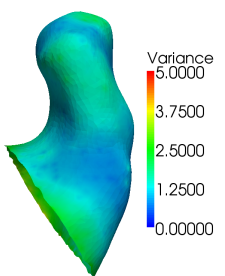

(a) [184 Shapes]

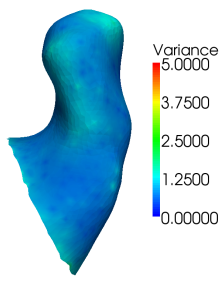

(b) [62 Shapes]

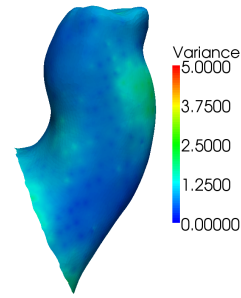

(d) [50 Shapes]

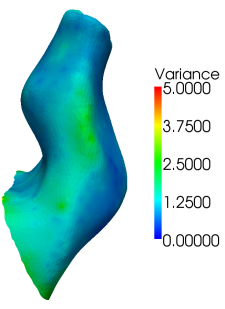

(c) [47 Shapes]

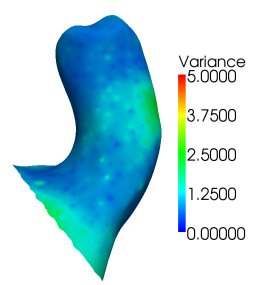

(e) $[25$ Shapes $]$

Figure 6. Left sided canal prototypes obtained by affinity propagation for (a) one cluster $\left(k_{1}=1\right)$, (b)-(e) four clusters $\left(k_{1}=4\right)$. The number of cluster members is shown in brackets. External ear parts are hidden.

In both cases, shape variations have mostly been captured at subregions of the parts where planes will eventually be placed. This is encouraging, since it indicates a correlated behavior between the characteristic shape information captured by the prototypes and the potential accuracy of inferred contours on new samples.

Fig. 8 presents inference examples of planes (planar contours) on different test shapes. For each part of a test shape we first find the best-matching prototype using Eqn. (5) followed by the plane propagation from the prototype to the test shape. It is required that normal angle deviations of the planes stay within a tolerance range of 15 degrees, and plane locations (the center of a planar contour) shall not deviate more than $3 \mathrm{~mm}$ from a given ground-truth position. The tolerance boundaries were specified by experts in the field of hearing aid design and are based on experience. Exceeding these tolerances will most likely lead to unfeasible surface modifications.

Orientation and location deviations are shown in Table 1. As can be observed, all estimated contours fulfilled the expert criteria described in the paragraph above. Finally, canal and external ear parts are modified independently, and

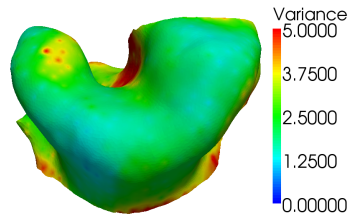

(a) [184 Shapes]

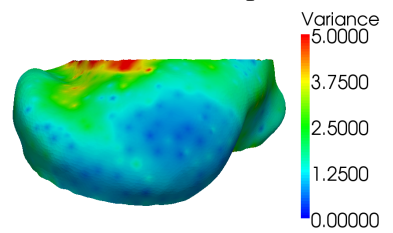

(c) [17 Shapes]

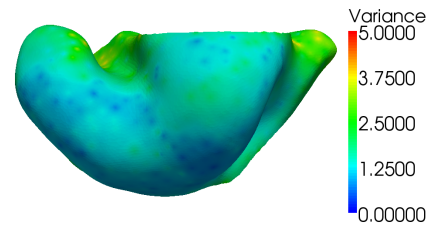

(e) [32 Shapes]

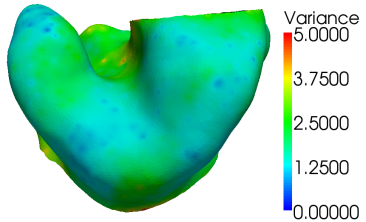

(b) [85 Shapes]

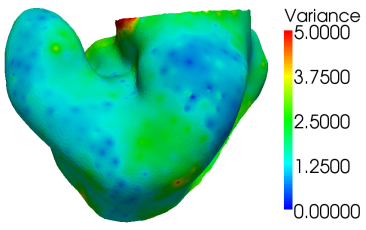

(d) [24 Shapes]

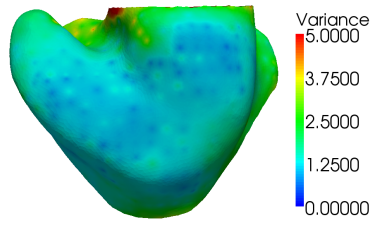

(f) [26 Shapes]
Figure 7. Left sided external ear prototypes obtained by affinity propagation for (a) one cluster $\left(k_{2}=1\right)$, and (b)-(f) five clusters $\left(k_{2}=5\right)$. The number of cluster members is shown in brackets. Canal parts are hidden.

the resulting meshes of test mesh number (4) and (8) are shown in Fig. 9.

The runtime of the prototype-based contour inference algorithm is dominated by the bipartite matching which can be solved in $O\left(N^{3}\right)$ time. In our current non-optimized implementation on a Pentium T2600/2 GHz, the estimation of a single contour on a test shape took about $5 \mathrm{~s}$. This includes the initial surface decomposition, finding the best-matching prototype for a part with approximately $N=700$ sample points and one iteration of point matching and affine transformation, and finally propagating the plane. It is an acceptable runtime for our application.

Table 1. Plane deviations for the test cases shown in Fig. 8

\begin{tabular}{|c|c|c|}
\hline Test case & Normal angle deviation & Location deviation \\
\hline$(1)$ & 8.5 degrees & $0.2 \mathrm{~mm}$ \\
\hline$(2)$ & 4.2 degrees & $0.4 \mathrm{~mm}$ \\
\hline$(3)$ & 9.5 degrees & $0.5 \mathrm{~mm}$ \\
\hline$(4)$ & 3.4 degrees & $0.8 \mathrm{~mm}$ \\
\hline$(5)$ & 3.1 degrees & $0.8 \mathrm{~mm}$ \\
\hline$(6)$ & 6.4 degrees & $0.7 \mathrm{~mm}$ \\
\hline$(7)$ & 5.7 degrees & $1.2 \mathrm{~mm}$ \\
\hline$(8)$ & 3.6 degrees & $0.2 \mathrm{~mm}$ \\
\hline
\end{tabular}



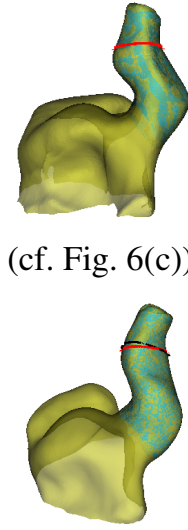

(1)
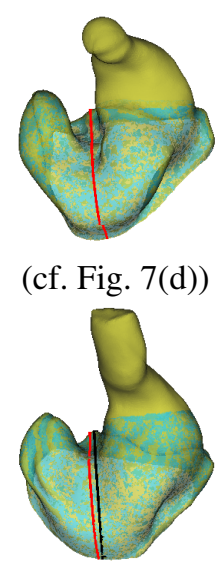

(5)

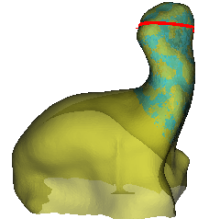

(cf. Fig. 6(b))

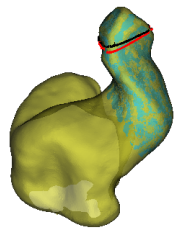

(2)

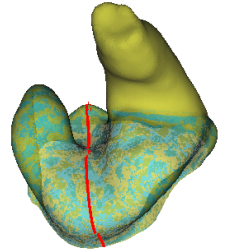

(cf. Fig. 7(b))

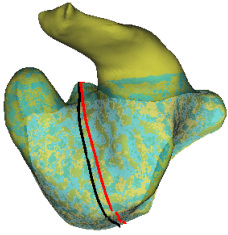

(6)

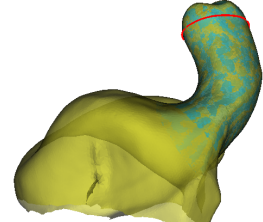

(cf. Fig. 6(e))

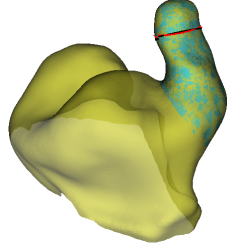

(3)

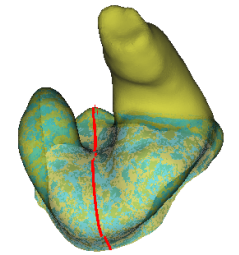

(cf. Fig. 7(b))

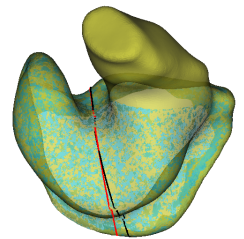

(7)

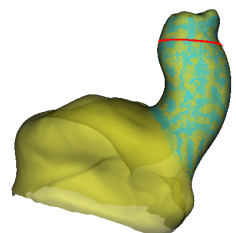

(cf. Fig. 6(d))

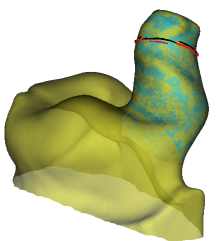

(4)

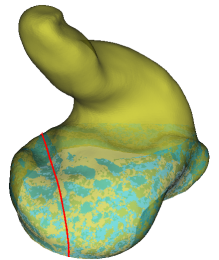

(cf. Fig. 7(c))

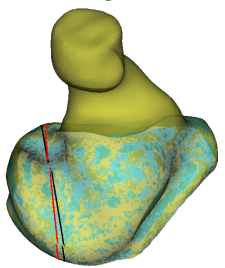

(8)

Figure 8. Prototype-based inference of planes (planar contours) on canal and external ear parts. The blue color corresponds to the part under consideration. The first row and the third row depict the best-matching part prototypes for the corresponding parts of the test meshes in the second and fourth row. The number in parenthesis indicates the test case. The red ground-truth contour was propagated from the part prototype to the test mesh below the prototype. An inferred contour is shown in black versus the ground-truth (red). The corresponding plane deviation values are presented in Table 1.

\section{Conclusions}

We have proposed a hierarchical framework that generalizes a digital design procedure for anatomical surface models in terms of a small number of prototypes. The latter have been derived from a code-book of anatomical parts together with operations that dictate how a shape undergoes modification. This allows inferences of operations on new instances according to their shape. We have demonstrated the utility of our approach through application to digital hearing aid manufacturing with promising results. A small number of prototypes were found to be sufficient to achieve an acceptable accuracy, while simultaneously increasing the efficiency.

While the initial results are promising, further investigation of the framework is needed. For example, it would be interesting to know how the accuracy of the cuts (con- tours) relative to the performance specification is affected by the choice of the number of clusters $k_{l}$. A second topic for future work is to extend the framework via a probabilistic inference approach of operations.

\section{References}

[1] G. Slabaugh, et al., "3D shape modeling for hearing aid design”, IEEE SPM, vol. 98, 2008.

[2] Y. Y. Cai, et al., "Simulation-based virtual prototyping of customized catheterization devices", Journal of Computing and Information Science in Engineering, vol. 4, 2004.

[3] S.-P. Sun, et. al., "Classification and mass production technique for three-quarter shoe insoles using non-weightbearing plantar shapes," Applied Ergonomics, 2008.

[4] K. W. Dalgarno, et al., "Mass customization of medical devices and implants: state of the art and future directions", 

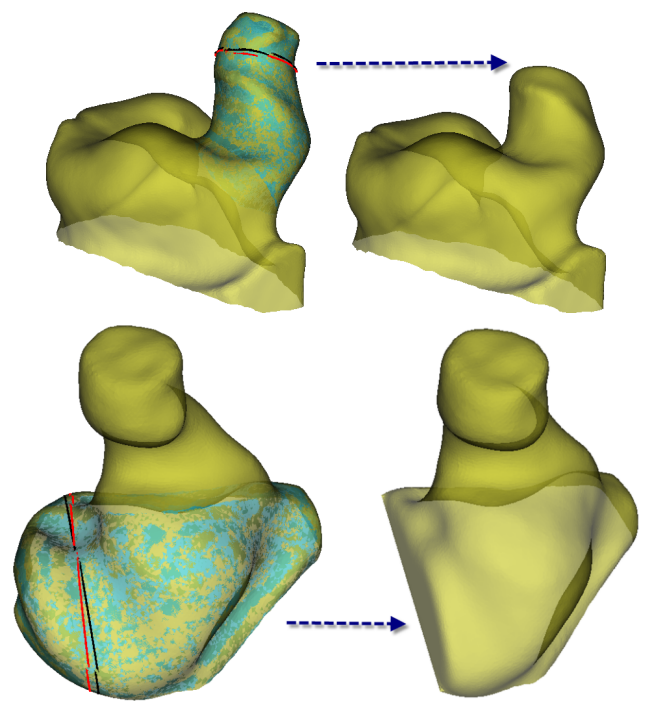

Figure 9. Surface modification for the test cases (4) and (8) using the propagated plane/planar contour (black color). The red contour represents the ground-truth. The blue colored partial surface (Left) depicts the part of interest in each case, i.e., canal part (Top) and external ear part (Bottom). Applying the operation yields the modified surface (Right). All surfaces are transparently rendered.

Virtual and Physical Prototyping, 2006.

[5] G. Unal, et al., "Customized design of hearing aids using statistical shape learning”, Proc. of MICCAI, 2008.

[6] C. Y. Ip, W. C. Regli, "A 3d object classifier for discriminating manufacturing processes", Computers and Graphics, 2006.

[7] P. J. Besl, N. D. McKay, "A method for registration of 3-d shapes", IEEE Trans. Pattern Analysis and Machine Intelligence, vol. 14, 1992.

[8] S. Belongie, et al., "Shape matching and object recognition using shape contexts", IEEE PAMI, vol. 24, no. 24, 2002.

[9] M. Koertgen, et al. "3D shape matching with $3 \mathrm{~d}$ shape contexts", Proceedings 7th Central European Seminar on Computer Graphics, 2003.

[10] A. E. Johnson, M. Hebert, "Using spin images for efficient object recognition in cluttered 3d scenes", IEEE Trans. PAMI, vol. 21, 1999.

[11] A. Frome, et al., "Recognizing objects in range data using regional point descriptors", Proceedings ECCV, vol. 3, 2004.

[12] B. Taati, M. Bondy, P. Jasiobedzki, M. Greenspan, "Variable Dimensional Local Shape Descriptors for Object Recognition in Range Data", ICCV, pp.1-8, 2007 IEEE 11th International Conference on Computer Vision, 2007.

[13] J. Feng, et. al., "Robust point correspondence matching and similarity measuring for 3D models by relative angle-context distributions", Image and Vision Computing, 2007.

[14] R. Jonker, A. Volgenant, "A shortest augmenting path algorithm for dense and sparse linear assignment problems", Computing, vol. 38, 1987.
[15] B. J. Frey, D. Dueck, "Clustering by passing messages between data points", Science, vol. 315, 2007.

[16] A. Zouhar, G. Unal, T. Fang, G. Slabaugh, H. Xie, F. McBagonluri, "Anatomically-aware, automatic, and fast registration of 3D ear impression models", Proceedings 3DPVT, 2006.

[17] http://www.nidcd.nih.gov/health/hearing/hearingaid.asp, National Institute on Deafness and Other Communication Disorders.

[18] http://www.geomagic.com/, Raindrop Geomagic. Geomagic studio: Reverse engineering and custom design software.

[19] http://www.innovmetric.com/, InnovMetrics. PolyWorks Inspection and modeling solutions.

[20] http://www.3shape.com/., 3Shape A/S. ShellDesigner, DentalDesigner, 3D Last. 